\title{
Implementation with renegotiation when preferences and feasible sets are state dependent
}

\author{
Luis Corchón - Matteo Triossi
}

\begin{abstract}
In this paper, we present a model of implementation where infeasible allocations are converted into feasible ones through a process of renegotiation that is represented by a reversion function. We describe the maximal set of Social Choice Correspondences that can be implemented in Nash Equilibrium in a class of reversion functions that punish agents for infeasibilities. This is used to study the implementation of the Walrasian Correspondence and several axiomatic solutions to problems of bargaining and taxation.
\end{abstract}

\section{Introduction}

Since Hurwicz's classic papers in the early 1970s, a great deal of attention has been devoted to the problem of implementing social choice rules when preferences are state dependent (see Jackson 2001 for a survey). In contrast, very few contributions have dealt with the problem of implementing social choice rules when the set of feasible outcomes is state dependent. The problem is that, in this case, some messages yield infeasible allocations, which requires that we describe how to deal with them. The standard approach is to design a series of mechanisms in which the planner can ex-post verify whether or not players are exaggerating individual endowments or

\footnotetext{
L. Corchón

Departmento de Economía, Universidad Carlos III, Calle Madrid 126, 28903 Getafe, Madrid, Spain e-mail: 1corchon@eco.uc3m.es

M. Triossi $(\bowtie)$

Centro de Economía Aplicada, Departamento de Ingeniería Industrial, Universidad de Chile, Avenida Republica 701, Santiago, Chile

e-mail: mtriossi@dii.uchile.cl
} 
technological capabilities (i.e., by asking them to put endowments on the table). ${ }^{1}$ If an infeasibility occurs, players expect serious punishment (Hurwicz et al. 1995; Tian 1993; Tian and Li 1995; Hong 1995, 1996, 1998; Serrano and Vohra 1997; Dagan et al. 1999). ${ }^{2}$

In this paper, we present a theory of how to deal with infeasibilities which is based on the idea that infeasible allocations are renegotiated. Consider the following example: The associate editor of a journal is in charge of a special issue for which she has selected 10 authors. She asks each author to submit a 20-page paper. One of the authors submits a 22-page paper and another author submits an 18-page one. In this case, it is likely that the editor will take no action. However, if both authors submit 22-page papers, she will have to deal with the infeasibility. It is likely that she will work with the authors to shorten the papers or with the managing editor in order to free up more pages, etc. In this case, feasibility is restored by means of negotiation. Another example is the legal system: once infeasibilities are detected, there are institutions designed to punish transgressors (if they can be identified) and to restore feasibility. ${ }^{3}$ Thus, the process used to deal with infeasibilities may reflect how agents renegotiate or how institutions operate. Furthermore, it is independent on the mechanism that created the infeasibility.

We model the social process that transforms infeasible allocations into feasible ones by means of a reversion function. This concept originates in Maskin and Moore (1999) and has been developed by Jackson and Palfrey (2001). In these papers, the reversion function formalizes the process of renegotiation through which agents trade goods allocated by the mechanism or veto some feasible allocations. In our case, the reversion function represents the way in which society reacts to infeasible allocations. Consequently, the properties that we impose on the reversion function are very different from those assumed by earlier literature.

For the purposes of this paper, we assume complete information. This is a clean scenario which looks to be a good candidate for a first trial of our approach. We focus on Nash implementation and assume that agents know the reversion function. Therefore, the reversion function induces new preferences, which we will call reverted preferences (this is the "translation principle" in Maskin and Moore (1999)). Reverted preferences are state dependent even if underlying preferences are not. Hence, when the feasible set is state dependent, implementation reduces to the case of implementation when only preferences are state dependent. However, as Maskin and Moore put it, "results from the standard literature are too abstract to give a clear indication of how serious a constraint renegotiation is...."

We focus our attention on a class of reversion function in which, should an infeasibility arise, at least one agent is made worse off. We call this a non-rewarding reversion

\footnotetext{
1 This assumption is called the "no exaggeration assumption." It implies that agents use different message spaces at different states of the world, see Hong (1998, p. 206, 11. 17-19).

2 The work on manipulation via endowments (Postlewaite 1979; Atlamaz and Klaus 2007) is also related to this literature.

${ }^{3}$ We can think of the feasible set including not only the properly feasible allocations, but also all punishments and additional devices that can be administered by the institutions designed, as well as the delays that may occur.
} 
function. Reversion functions considered by the previous literature on renegotiation do not fall into this class because they assume that agents are made better off by renegotiating. The difference is explained by the fact that in their case, renegotiation comes from the inability of the mechanism to stop agents from reaching mutually beneficial trades. In our case, renegotiation arises from the physical impossibility of carrying out the intended plans so that somebody has to make a sacrifice in order to achieve feasibility. The class of non-rewarding reversion functions is too wide, though. Given the novelty of our approach, we think that to look for a characterization of implementable social choice rules under this class is a too ambitious task. A more modest research strategy is to charter the boundaries of implementable social choice rules when reversion functions are non-rewarding. We do this by introducing an extreme case of non-rewarding reversion function where, should an infeasibility arise, all agents are punished so that they strictly prefer any allocation without punishment to the situation in which they are punished. This strong form of punishment, which we will call generalized severe, resembles the one implicitly assumed by the previous literature. In our case it serves an instrumental role: it provides necessary conditions for the implementation of social choice rules if the reversion function is generalized severe (Proposition 1). Thus, generalized severe reversion functions may be helpful to direct further research on this topic because they are manageable and if a social choice rule is non implementable with a generalized severe reversion function it is not implementable under any non-rewarding reversion function.

A simple adaptation of the classic results shows that monotonicity is a necessary and almost sufficient condition for implementation in Nash equilibrium when reverted preferences are given by the generalized severe reversion function (Remark 1). Thus, our first task is to characterize monotonicity. We show that it is equivalent to a weak form of individual rationality and a generalized form of contraction consistency (Proposition 2). The former property is satisfied by most social choice rules and the latter is similar to Nash independence of irrelevant alternatives.

Next, we apply the previous result to several frameworks and compare our findings with the earlier literature. We begin by considering exchange economies. Here, the non-rewarding condition may be violated unless renegotiation is sufficiently costly. In this case, the non-rewarding condition can be considered as a simplification that narrows down the class of renegotiation functions and thus is useful for obtaining analytical results. We prove that in these environments, weak unanimity is trivially satisfied by any individually rational social choice rule. But the individual rationality requirement, which is necessary and sufficient for feasible implementation in the set-up considered by Hurwicz et al. (1995), is not necessary nor sufficient for implementation in our framework. This is due to the fact that in our case a generalized form of contraction consistency must be satisfied as well. We show that the Constrained Walrasian Rule satisfies generalized contraction consistency and is thus implementable (Proposition 3). Next, we turn our attention to bargaining problems. We show that the Nash Bargaining solution can be implemented if the disagreement point is not state dependent (Proposition 4). This agrees with the findings obtained by Serrano and Vohra (1997) and Naeve (1999) in a different framework. We also show that the KalaiSmorodinski solution is not implementable. Finally, we consider the taxation problem in which the mechanism must collect a given amount of taxes. We describe the taxation 
rules that can be implemented and find that any taxation rule which continuously varies on incomes whenever incomes are larger than proposed taxes cannot be implemented in our framework. This contrasts with a result by Dagan et al. (1999) in which, assuming that agents cannot exaggerate anyone's income, they are able to implement any taxation method. The difference between our approaches is that the no exaggeration assumption in their case rules out deviations that are possible in our model.

In the sections that follow, we describe the model (Sect. 2), introduce reversion functions (Sect. 3) and study implementation under the assumption that the reversion function is non-rewarding (Sects. 4, 5) before presenting our conclusions (Sect. 6).

\section{The model}

In this section we provide the main definitions. Let us first describe the environment. Let $I=\{1, \ldots, n\}$ be the set of agents. Let $\omega_{i}$ denote agent $i$ 's type and let $\Omega_{i}$ denote agent $i$ 's type set. Let $\Omega \subseteq \prod_{i=1}^{n} \Omega_{i}$ be the set of all possible states of the world. Each $\omega \in \Omega$ yields a feasible set $A(\omega)$ and a preference profile $R(\omega)=\left(R_{1}(\omega), \ldots, R_{n}(\omega)\right)$. The feasible set $A(\omega)$ contains all feasible allocations including punishments that arise at state $\omega$. Set $A \equiv \bigcup_{\omega \in \Omega} A(\omega) . R_{i}(\omega)$ is a preference relation, a complete, reflexive and transitive binary relation on $A(\omega) . P_{i}(\omega)$ denotes the corresponding strict preference relation. Let $L_{i}(a, \omega)=\left\{x \in A(\omega): a R_{i}(\omega) x\right\}$ be agent $i$ 's lower contour set of $a$ at $\omega$.

A correspondence $F: \Omega \rightarrow A$ such that $F(\omega) \subseteq A(\omega)$ for all $\omega \in \Omega$ will be called a Social Choice Rule (SCR for brevity). A mechanism is a pair $(M, g)$ where $M \equiv \prod_{i=1}^{n} M_{i}$ is the message space and $g: M \rightarrow A$ is the outcome function. $M_{i}$ denotes agent $i$ 's message space. Let $m=\left(m_{1}, . ., m_{n}\right) \in M$ be a list of messages, also written $\left(m_{i}, m_{-i}\right)$. Given $\omega \in \Omega$, a mechanism $(M, g)$ induces a game $(M, g, R(\omega))$. A message profile $m^{*} \in M$ is a Nash equilibrium for $(M, g, R(\omega))$ if, for all $i \in I, g\left(m^{*}\right) R_{i}(\omega) g\left(m_{i}, m_{-i}^{*}\right)$ for all $m_{i} \in M_{i}$. NE $(M, g, R(\omega))$ will denote the set of Nash equilibrium outcomes of $(M, g, R(\omega))$. The mechanism $(M, g)$ implements $F$ in Nash equilibrium if $\operatorname{NE}(M, g, R(\omega))=F(\omega)$ for all $\omega \in \Omega$.

\section{Reversion functions}

Since outcomes that are feasible in some states may be infeasible in others, we must describe how society deals with infeasible allocations. We assume that if an allocation is infeasible, it is transformed into a feasible one. The systematic way in which the reallocation process takes place will be called a reversion function. ${ }^{4}$ This reallocation may correspond to a "free-market renegotiation" or to a process where an institution applies some rule, i.e., a rationing scheme, a bankruptcy rule, penalties to some individuals, etc. Formally:

Definition 1 A reversion function is a map $h: A \times \Omega \rightarrow A$ such that, for every $\omega \in \Omega$ : (i) $h(a, \omega) \in A(\omega)$ for all $a \in A$ and (ii) $h(a, \omega)=a$ for all $a \in A(\omega)$.

\footnotetext{
${ }^{4}$ See Amorós (2004) for a model with several reversion functions.
} 
A reversion function always yields feasible allocations (condition (i) above) and feasible allocations are not renegotiated (condition (ii) above). The latter condition is postulated in order to separate the issue of mutually advantageous renegotiation, which was the focus of the previous literature, from the issue of infeasibility, which is the focus of this paper. ${ }^{5}$ In other words, this condition allows us to analyze renegotiation caused by infeasibility alone.

Under weak conditions, if the reversion function can be chosen by the planner, any single valued SCR can be implemented (a proof is available upon request). But the designer, by assumption, cannot condition her actions on the state of the world.

To explain the next concept, consider the simplest possible case: at states of the world $\omega$ and $\omega^{\prime}$ the preference profiles are the same, say $R$. Let $a, b$, and $c$ be three allocations that are feasible at state $\omega$. Assume that $a P_{i} b P_{i} c$ for some agent $i$. Allocation $a$ is not feasible at state $\omega^{\prime}$ and is renegotiated to $c$, allocation $b$ is feasible at $\omega^{\prime}$. So, even if the underlying preferences are the same in both states, player $i$ prefers $a$ to $b$ at state $\omega$ and $b$ to $a$ at $\omega^{\prime}$. In order to formalize and extend this idea, we offer the following definition.

Definition 2 Given $\omega \in \Omega$ and a reversion function $h$, the reversion of $R(\omega)$ on $A(\omega)$, denoted by $R^{h}(\omega)$, is defined by:

$$
a R_{i}^{h}(\omega) b \Longleftrightarrow h(a, \omega) R_{i}(\omega) h(b, \omega), \text { for all } a, b \in A, \text { for all } i \in I \text {. }
$$

Then, when the reversion function is $h$, we can interpret that agents' preferences are the reverted preferences, i.e., they only care about reverted allocations. Let $L_{i}^{h}(a, \omega)=$ $\left\{b \in A: h(a, \omega) R_{i}(\omega) h(b, \omega)\right\}$ be the lower contour set of $a$ at $\omega$ with respect to $R_{i}^{h}(\omega)$.

The next definition is a straightforward adaptation of the standard notion of implementation in Nash equilibrium.

Definition 3 A SCR $F$ is $h$-implementable in Nash Equilibrium if there exists a game form $(M, g)$ such that, for all $\omega \in \Omega$ :

$$
F(\omega)=h\left(\mathrm{NE}\left(M, g, R^{h}(\omega)\right), \omega\right)
$$

In words, $F$ is $h$-implementable in Nash equilibrium if and only if it is implementable in Nash equilibrium when, for each $\omega \in \Omega$, the correspondent preference profile is $R^{h}(\omega)$. Once we consider that agents' preferences are induced by the reversion function, we can deal with $h$-implementation exactly in the same way as in the classical implementation problem.

When considering the restrictions that a state dependent feasible set imposes on implementation, we concentrate on monotonicity (or Maskin monotonicity). Monotonicity is a necessary and almost sufficient condition for a SCR to be implementable in Nash equilibrium (see Maskin and Moore 1999; Repullo 1987; Jackson 2001). It is therefore the first condition to be addressed. When a SCR satisfies monotonicity,

5 A tautological interpretation is that $A(\omega)$ is the set of allocations that are not renegotiated at $\omega$. 
if an alternative is implemented at one state of the world and rises in every agent's preference ranking at another state of the world, then it must be implemented also at the second state. Now we restate the definition of monotonicity in terms of reverted preferences. Let $h$ be a reversion function.

Definition 4 A social choice rule $F$ is h-monotonic if, for all $\omega, \omega^{\prime} \in \Omega$ and for every $a \in A$ such that $h(a, \omega) \in F(\omega)$

$$
L_{i}^{h}(a, \omega) \subseteq L_{i}^{h}\left(a, \omega^{\prime}\right) \text { for all } i \Longrightarrow h\left(a, \omega^{\prime}\right) \in F\left(\omega^{\prime}\right)
$$

Similarly, a SCR $F$ that satisfies $h$-no veto power must select an allocation which is at the top of the reverted preference ranking of all agents except at most one. The importance of these concepts is highlighted by the following remark, whose proof is a straightforward adaptation of a standard result mentioned before and is therefore omitted:

Remark 1 If a social choice rule is $h$-implementable in Nash equilibrium, it is $h$-monotonic. Moreover, in environments in which \#I>2 if a social choice rule is $h$-monotonic and satisfies $h$-no veto power, it is $h$-implementable in Nash equilibrium.

\section{Non-rewarding reversion functions: basic results}

In principle, the reversion function can take any form which makes difficult to characterize which SCR can be implemented (i.e., to check $h$-monotonicity). Thus, we need to restrict the possibilities opened by renegotiation. Since we assumed that only infeasible allocations are renegotiated, it seems natural to assume that renegotiation cannot be advantageous for all players. We will formalize this idea by the concept of non-rewarding reversion function. This concept is discussed in detail after we present a formal definition below. Clearly, this is not the only possibility to reduce the set of reversion functions and our approach is only a first cut at the problem. We show that a necessary condition for a SCR to be $h$-implementable when $h$ is non-rewarding is that this SCR is $h$-implementable when the reversion function takes a particularly simple form. It is the case where, should an infeasibility arise, the reversion achieves the worst possible allocation. We will call the latter generalized severe. Having simplified the problem to the point in which we have singled out a unique reversion function, we characterize $h$-implementable SCR when $h$ is generalized severe. Thus, we provide necessary conditions for $h$-implementation under non-rewarding reversion functions. ${ }^{6}$

Definition 5 A reversion function $h$ is non-rewarding if, for all $\omega, \omega^{\prime} \in \Omega$ and all $a \in A(\omega)$ :

\footnotetext{
6 It may be argued that a generalized severe reversion does not fit well with the idea of renegotiation. But recall that generalized severe reversion is just a way to show which SCR might be implemented under non-rewarding renegotiation and not a realistic description of how renegotiation is conducted. A comparison with the revelation principle may be useful. Direct mechanisms are used not because they are realistic or have good properties but because they provide necessary conditions for the existence of implementing mechanisms and thus they are helpful to clear the field.
} 
(i) if $a \notin A\left(\omega^{\prime}\right)$ there exists $i \in I \operatorname{such} L_{i}^{h}(a, \omega) \nsubseteq L_{i}^{h}\left(a, \omega^{\prime}\right)$.

(ii) if there exist $i \in I$ and $b \in A$ with $a R_{i}(\omega) h(b, \omega)$ and $h\left(b, \omega^{\prime}\right) P_{i}\left(\omega^{\prime}\right) h\left(a, \omega^{\prime}\right)$ then there exist $j$ and $c \in A\left(\omega^{\prime}\right)$ such that $a R_{j}(\omega) h(c, \omega)$ with $c P_{j}\left(\omega^{\prime}\right) h\left(a, \omega^{\prime}\right)$.

The first condition asserts that if an allocation $a$ passes from being feasible at state $\omega$ to being infeasible at state $\omega^{\prime}$ not all agents improve their positions. Notice that it does not imply that anybody is punished for the infeasibility. The change of $a$ in the ranking when passing from state $\omega$ to state $\omega^{\prime}$ might simply reflect a shift in the underlying preferences like in the theory of implementation with a fixed feasible set. The second condition takes care of this aspect. It implies that if there is a preference reversal around an allocation $a$ which is feasible at $\omega$ and it does not reflects a shift in the underlying preferences (the case where $b \in A(\omega) \cap A\left(\omega^{\prime}\right)$ ), then somebody has actually to pay the cost of the renegotiation passing from state $\omega^{\prime}$ to state $\omega$ : an allocation $c \in A\left(\omega^{\prime}\right)$ which was strictly better than $a$ at $\omega^{\prime}$ in some agent's preferences, is renegotiated to an allocation which belongs to the lower contour set of $a$ at $\omega$, with respect to the preferences of the same agent. In particular, conditions (i) and (ii) together imply that when an allocation passes from being feasible at state $\omega$, to being infeasible at $\omega^{\prime}$ at least one agent suffers as a consequence of infeasibility in a way that could have been accomplished through a feasible allocation (for instance, the agent who is deemed responsible for the infeasibility is punished).

Another interpretation of condition (ii) is the following. If $a$ is feasible at state $\omega$ the strict upper contour set cannot be larger for every agent when passing from state $\omega^{\prime}$ to state $\omega$, unless this enlargement depends on a change in the underlying preferences.

Conditions (i) and (ii) are independent. It is easy to write an example where (ii) holds and (i) does not. ${ }^{7}$ Next, we present an example where (ii) holds and (i) does not. This example will be helpful in explaining condition (ii).

Example 1 There are two states of the world $\omega$ and $\omega^{\prime}$ and two agents $i=1,2$. The feasible sets are $A(\omega)=\left\{a_{1}, a_{2}, a_{3}, a_{4}, a_{5}\right\}$ and $A\left(\omega^{\prime}\right)=\left\{a_{1}, a_{2}^{\prime}, a_{3}, a_{4}, a_{5}\right\}$. Preferences for agent 1 are $a_{5} P_{1}(\omega) a_{1} P_{1}(\omega) a_{2} P_{1}(\omega) a_{4} P_{1}(\omega) a_{3}$ and $a_{5} P_{1}\left(\omega^{\prime}\right) a_{2}^{\prime} P_{1}$ $\left(\omega^{\prime}\right) a_{1} P_{1}\left(\omega^{\prime}\right) a_{4} P_{1}\left(\omega^{\prime}\right) a_{3}$. Preferences for agent 2 are $a_{3} P_{2}(\omega) a_{5} P_{2}(\omega) a_{1} P_{2}(\omega)$ $a_{2} P_{2}(\omega) a_{4}$ and $a_{5} P_{2}\left(\omega^{\prime}\right) a_{1} P_{2}\left(\omega^{\prime}\right) a_{4} P_{2}\left(\omega^{\prime}\right) a_{2}^{\prime} P_{2}\left(\omega^{\prime}\right) a_{3}$. The reversion function is $h\left(a_{2}, \omega^{\prime}\right)=a_{2}^{\prime}$ and $h\left(a_{2}^{\prime}, \omega\right)=a_{5}$. This reversion function satisfies condition (i) in Definition 5. Indeed, $a_{2} P_{2}^{h}(\omega) a_{4}, a_{4} P_{2}^{h}\left(\omega^{\prime}\right) a_{2}$ and $a_{2}^{\prime} P_{2}^{h}\left(\omega^{\prime}\right) a_{3}, a_{3} P_{2}^{h}(\omega)$ $a_{2}^{\prime}$. However, it does not satisfy condition (ii) in Definition 5. Indeed, $a_{1} P_{1}^{h}(\omega) a_{2}$ and $a_{2} P_{2}^{h}\left(\omega^{\prime}\right) a_{1}$ but there is no $c \in A\left(\omega^{\prime}\right)$ such that $c P_{i}^{h}\left(\omega^{\prime}\right) a_{1}$ and $a_{1} P_{i}^{h}(\omega) c$ for some $i \in\{1,2\}$ because $h\left(a_{2}^{\prime}, \omega\right)=a_{5} P_{i}(\omega) a_{1}$ for $i=1,2$.

In Example 1, $a_{2}$ goes up in agent's 1 ranking with respect to $a_{1}$ passing from state $\omega$ to state $\omega^{\prime}$. Condition (i) is satisfied because $a_{2}$ change her position with respect to $a_{4}$ passing from state $\omega$ to state $\omega^{\prime}$ and $a_{2}^{\prime}$ change its position with respect to $a_{3}$ passing from state $\omega^{\prime}$ to state $\omega$. This change only reflects a change in the underlying preferences. Notice that allocation $a_{5}$ is preferred to $a_{1}$ by both agents at state $\omega$ and at state $\omega^{\prime}$. Allocation $a_{2}^{\prime}$ is better that $a_{1}$ at state $\omega^{\prime}$ in the preferences of agent 1 but

7 Available upon request. 
it not feasible at state $\omega$. Allocation $a_{2}^{\prime}$ is renegotiated to allocation $a_{5}$ at state $\omega$ so condition (ii) does not hold.

Notice that there is nothing pathological about the renegotiation function used in Example 1. This leads us to think that the second part of Definition 5 indeed has a bite. However, we will see in next section that there are natural renegotiation functions that fulfill this condition.

Consider now a specific family of reversion functions: suppose that, should an infeasibility arise, players are redirected to what they consider to be the worst possible allocation. This reversion function resembles the (implicit) assumption made in previous papers that agents do not choose infeasible messages because the planner detects infeasibility and imposes a punishment in such a way that agents strictly prefer any other feasible allocation to this punishment. However, our interest in this particular reversion function arises from the fact that it allows us to find the maximal set of SCR that can be implemented under non-rewarding renegotiation (see Proposition 1). Let $G \in A(\omega)$ for all $\omega \in \Omega$ be such that $a P_{i}(\omega) G$ for all $i$, for all $a \in A(\omega) \backslash\{G\}$. We assume that, for all $\omega \in \Omega$, the feasible set $A(\omega)$ contains at least one allocation different from $G$. $G$ will be called the generalized punishment point because all agents are penalized.

Definition 6 A reversion function is generalized severe if $s(a, \omega)=G$ if $a \notin A(\omega)$.

The induced preferences will be denoted by $R^{s}(\omega)$. Preferences $R^{s}$ are characterized by the following three properties:

(1) If $a, b \in A(\omega)$ then $a R_{i}^{s}(\omega) b$ if and only if $a R_{i}(\omega) b$ for all $i \in I$.

(2) If $a \in A(\omega)$ with $a \neq G$ and $b \notin A(\omega)$ then $a P_{i}^{s}(\omega) b$ for all $i \in I$.

(3) If $a, b \notin A(\omega)$ then $a I_{i}^{s}(\omega) b$ for all $i \in I$.

We assume that the planner never wants to implement alternative $G$. Formally, we consider only social choice rules $F$ such that $G \notin F(\omega)$ for all $\omega \in \Omega$.

A generalized severe reversion function punishes players for the implementation of infeasible allocations. A rewarding reversion function can provide the opposite incentives. There exist SCR that are implementable using a rewarding $h$ but not using a generalized severe reversion function. Let $\Omega=\left\{\omega, \omega^{\prime}\right\}, A(\omega)=\{a, b, c, G\}$ and $A\left(\omega^{\prime}\right)=\{a, b, G\}$. Let $n=2$ and $R_{i}(\omega)=R_{i}\left(\omega^{\prime}\right)=R$ for $i=1,2$ where $b P a P c$. Let $F(\omega)=a$ and $F\left(\omega^{\prime}\right)=b$. Let $h\left(c, \omega^{\prime}\right)=b$. The function $h$ does not satisfy the non-rewarding assumption at $c . F$ is $h$-implementable in NE by the simple mechanism where agent 1 chooses among $a$ and $c$, but it cannot be implemented with a generalized severe reversion function because $F$ is not monotonic with respect to preferences $R^{s}$.

We show that generalized severe reversion functions implement the largest set of social choice rules among the class of non-rewarding reversion functions where $h$-no veto power holds.

Proposition 1 Let $F$ be an h-monotonic SCR. If $h$ is non-rewarding, then F is monotonic with a generalized severe reversion function. Thus, if $F$ satisfies $h$-no veto power, it is implementable in Nash Equilibrium with a generalized severe reversion function.

Proof In the proof $s$ denotes the generalized severe reversion function. Let $a \in F(\omega)$ and assume that for some $\omega^{\prime}, L_{i}^{S}(a, \omega) \subseteq L_{i}^{s}\left(a, \omega^{\prime}\right)$ for every $i \in I$. First, we show that 
$a \in A\left(\omega^{\prime}\right)$. We prove the claim by contradiction: assume that $a \notin A\left(\omega^{\prime}\right)$. In this case, $L_{i}^{s}\left(a, \omega^{\prime}\right)=\{G\} \cup A \backslash A\left(\omega^{\prime}\right)$, so $L_{i}^{s}(a, \omega)=\left(L_{i}(a, \omega) \cap A(\omega)\right) \cup A \backslash A(\omega) \subseteq\{G\} \cup$ $A \backslash A\left(\omega^{\prime}\right)$. It follows that $A \backslash A(\omega) \subseteq A \backslash A\left(\omega^{\prime}\right)$ so $A\left(\omega^{\prime}\right) \subseteq A(\omega)$. The reversion function $h$ is non-rewarding and $a \notin A\left(\omega^{\prime}\right)$, so $L_{i}^{h}(a, \omega) \nsubseteq L_{i}^{h}\left(a, \omega^{\prime}\right)$. Then, there exists $b$ such that $a R_{i}(\omega) h(b, \omega)$ and $h\left(b, \omega^{\prime}\right) P\left(\omega^{\prime}\right) a$. From the definition of a nonrewarding reversion function there exist $j$ and $c \in A\left(\omega^{\prime}\right)$ such that $a R_{j}(\omega) h(c, \omega)$ and $c P_{j}\left(\omega^{\prime}\right) h\left(a, \omega^{\prime}\right)$, but $h(c, \omega)=c$, because $A\left(\omega^{\prime}\right) \subseteq A(\omega)$, which yields a contradiction because $L_{j}^{s}(a, \omega) \subseteq L_{j}^{s}\left(a, \omega^{\prime}\right)$. Then it must be the case that $a \in A\left(\omega^{\prime}\right)$. In order to complete the proof it suffices to show that $L_{i}^{h}(a, \omega) \subseteq L_{i}^{h}\left(a, \omega^{\prime}\right)$ for every $i \in I$. We prove the claim by contradiction: assume that there exist $i \in I$ and $b \in A$ such that $a R_{i}(\omega) h(b, \omega)$ and $h(b, \omega) P_{i}\left(\omega^{\prime}\right) a$. From the definition of a non-rewarding reversion function there exist $j$ and $c \in A\left(\omega^{\prime}\right)$ such that $a R_{j}(\omega) h(c, \omega)$ with $c P_{j}\left(\omega^{\prime}\right) h\left(a, \omega^{\prime}\right)$. If $c \in A(\omega)$ then $h(c, \omega)=c \in L_{j}^{s}(a, \omega) \backslash L_{j}^{s}\left(a, \omega^{\prime}\right)$, a contradiction. If $c \notin A(\omega)$ then $s(c, \omega)=G$ and $c \in L_{j}^{s}(a, \omega) \backslash L_{j}^{s}\left(a, \omega^{\prime}\right)$, a contradiction. The last claim follows because $h$-no veto power implies no veto power with a generalized severe reversion function.

The reverse of Proposition 1 does not hold. There exist non-rewarding $h$ and SCR $F$ such that $F$ is monotonic under generalized severe reversion but not $h$-monotonic. Let $\Omega=\left\{\omega, \omega^{\prime}\right\}, A\left(\omega^{\prime}\right)=\{a, b, c, d, e, G\}$ and $A(\omega)=\{a, c, d, e, G\}$. Let $n=2$. Let $d P_{1}(\omega) c P_{1}(\omega) a P_{1}(\omega) e$ and let $d P_{1}\left(\omega^{\prime}\right) c P_{1}\left(\omega^{\prime}\right) b P_{1}\left(\omega^{\prime}\right) a P_{1}(\omega) e$. Let $e P_{2}(\omega) c P_{2}(\omega) d P_{2}(\omega) a$ and let $b P_{2}\left(\omega^{\prime}\right) a P_{2}\left(\omega^{\prime}\right) c P_{2}\left(\omega^{\prime}\right) e P_{2}\left(\omega^{\prime}\right) d$. Let $h(b, \omega)=d$. The reversion function $h$ is non-rewarding. Let $f$ defined by $f(\omega)=$ $\{a\}, f\left(\omega^{\prime}\right)=\{b\}$. The social choice function $f$ is implementable in Nash equilibrium under generalized severe reversion by the simple mechanism where agent 1 chooses among $a$ and $b$. However $L_{1}^{h}(a, \omega)=L_{1}^{h}\left(a, \omega^{\prime}\right)=\{a, e, G\}, L_{2}^{h}(a, \omega)=$ $\{a, e, G\} \subseteq\{a, d, e, G\}=L_{2}^{h}\left(a, \omega^{\prime}\right)$, so $f$ is not $h$-monotonic. In the example $L_{i}^{h}(a, \omega) \subseteq L_{i}^{h}\left(a, \omega^{\prime}\right)$ for $i=1,2$, but $L_{1}^{s}(a, \omega) \nsubseteq L_{1}^{s}\left(a, \omega^{\prime}\right)$. Indeed, $b \in A\left(\omega^{\prime}\right) \backslash A(\omega)$ and $b P_{1}\left(\omega^{\prime}\right) a$ so $b$ can be used to prevent the implementation of $a$ at state $\omega^{\prime}$ when the generalized severe reversion function is used. However, the same $b$ cannot be used to prevent the implementation of $f$ at $\omega^{\prime}$ if $h$ is used because $h(b, \omega)=d$ so $b \notin L_{i}^{h}(a, \omega)$ for $i=1,2$.

We now introduce two properties that are necessary and sufficient for $h$-monotonicity under generalized severe reversion.

Definition 7 A SCR $F$ satisfies Weak Unanimity (WU) if, for all $\omega, \omega^{\prime} \in \Omega$ such that $A\left(\omega^{\prime}\right) \subseteq A(\omega)$ and for all $a \in A(\omega) \backslash A\left(\omega^{\prime}\right)$ such that $L_{i}(a, \omega) \subseteq\{G\} \cup A \backslash A\left(\omega^{\prime}\right)$ for all $i \in I, a \notin F(\omega)$.

Assume that all alternatives that are available at $\omega^{\prime}$ are available at $\omega$, too. Let $a$ be available at $\omega$ but not at $\omega^{\prime}$. WU prescribes that if all alternatives that are available at $\omega^{\prime}$ are strictly better than $a$ at state $\omega$ for all agents, the planner should not choose to implement $a$ at state $\omega$. Breaking WU would create a problem of coordination at state $\omega^{\prime}$ : if such an $a$ was chosen at state $\omega$, any Nash equilibrium yielding $a$ at $\omega$ would be a Nash equilibrium at $\omega^{\prime}$ too, yielding $h\left(a, \omega^{\prime}\right)=G$.

Notice that WU is equivalent to the following condition: if $A\left(\omega^{\prime}\right) \subseteq A(\omega)$ and $a \in F(\omega) \backslash A\left(\omega^{\prime}\right)$ then there exists $b \in A\left(\omega^{\prime}\right)$, such that $a R_{i}(\omega) b$ for some $i \in I$. If 
WU holds, such a $b$ can be used to prevent the implementation of $G$ at state $\omega^{\prime}$. When the feasible set does not depend on the state of the world WU holds emptily.

Definition 8 A SCR $F$ satisfies Generalized Contraction Consistency (GCC) if, for all $\omega, \omega^{\prime} \in \Omega$, and for all $a \in F(\omega) \cap A\left(\omega^{\prime}\right)$ such that $L_{i}(a, \omega) \cap A\left(\omega^{\prime}\right) \subseteq L_{i}\left(a, \omega^{\prime}\right)$ and $A\left(\omega^{\prime}\right) \backslash A(\omega) \subseteq L_{i}\left(a, \omega^{\prime}\right)$ for all $i \in I, a \in F\left(\omega^{\prime}\right)$.

When preferences are fixed and $A\left(\omega^{\prime}\right) \subseteq A(\omega), A\left(\omega^{\prime}\right) \backslash A(\omega)=\varnothing \subseteq L_{i}(a, \omega)$ for all $i$. In such a case, GCC prescribes choosing at state $\omega^{\prime}$ any feasible allocation we have chosen at $\omega$. Thus, GCC is a weak version of Nash Independence of Irrelevant Alternatives (see Roemer 1996, p. 55). When the feasible set does not depend on the state of the world GCC coincides with Maskin Monotonicity. In the general case, GCC says that if $a$ is selected at state $\omega$, is feasible at $\omega^{\prime}$, rises in everybody's preference ranking with respect to the alternatives that are feasible at $\omega^{\prime}$ only and no better alternatives are available in $A\left(\omega^{\prime}\right) \backslash A(\omega)$, then $a$ must be selected also at $\omega^{\prime}$.

Proposition 2 A SCR is monotonic under generalized severe reversion if and only if it satisfies Weak Unanimity and Generalized Contraction Consistency.

Proof (Necessity) In the proof $s$ denotes the generalized severe reversion function. We first show the necessity of WU. Let $A\left(\omega^{\prime}\right) \subseteq A(\omega)$ and let $a \in F(\omega) \backslash A\left(\omega^{\prime}\right)$. The proof is by contradiction. Assume that $L_{i}(a, \omega) \subseteq\{G\} \cup A \backslash A\left(\omega^{\prime}\right)$ for all $i$. Observe that $L_{i}^{s}\left(a, \omega^{\prime}\right)=\{G\} \cup A \backslash A\left(\omega^{\prime}\right)$ for all $i$. We can write $L_{i}^{s}(a, \omega)=$ $L_{i}(a, \omega) \cap A(\omega) \cup A \backslash A(\omega)$. From the hypothesis of contradiction $L_{i}^{s}(a, \omega) \subseteq$ $\{G\} \cup A \backslash A\left(\omega^{\prime}\right) \cup A \backslash A(\omega)$ for all $i$. Since $A\left(\omega^{\prime}\right) \subseteq A(\omega)$ then $A \backslash A(\omega) \subseteq A \backslash A\left(\omega^{\prime}\right)$. It follows that $L_{i}^{s}(a, \omega) \subseteq\{G\} \cup A \backslash A\left(\omega^{\prime}\right)=L_{i}^{s}\left(a, \omega^{\prime}\right)$ for all $i$. As $F$ satisfies $s$-monotonicity, $s\left(a, \omega^{\prime}\right)=G \in F\left(\omega^{\prime}\right)$, which yields a contradiction. Now consider GCC. Let $a \in F(\omega) \cap A\left(\omega^{\prime}\right)$. Assume that $L_{i}(a, \omega) \cap A\left(\omega^{\prime}\right) \subseteq L_{i}\left(a, \omega^{\prime}\right)$ and that $A\left(\omega^{\prime}\right) \backslash A(\omega) \subseteq L_{i}\left(a, \omega^{\prime}\right)$ for all $i \in I$. We next prove that $L_{i}^{s}(a, \omega) \subseteq$ $L_{i}^{s}\left(a, \omega^{\prime}\right)$ for all $i$. Observe that both $L_{i}(a, \omega) \cap A\left(\omega^{\prime}\right)$ and $A\left(\omega^{\prime}\right) \backslash A(\omega)$ are subsets of the lower contour set of $a$ at $\omega$. We can decompose $L_{i}^{s}(a, \omega)$ as follows: $L_{i}^{s}(a, \omega)=L_{i}(a, \omega) \cap A\left(\omega^{\prime}\right) \cup\left[L_{i}(a, \omega) \cap A(\omega)\right] \backslash A\left(\omega^{\prime}\right) \cup A \backslash A(\omega)$. Then $L_{i}^{s}(a, \omega) \subseteq L_{i}\left(a, \omega^{\prime}\right) \cap A\left(\omega^{\prime}\right) \cup A \backslash A\left(\omega^{\prime}\right) \cup A \backslash A(\omega) \subseteq L_{i}^{s}\left(a, \omega^{\prime}\right) \cup A \backslash A(\omega)$ for all $i$. Observe that $A \backslash A(\omega)=A\left(\omega^{\prime}\right) \backslash A(\omega) \cup\left[A \backslash A\left(\omega^{\prime}\right)\right] \backslash A(\omega)$. From the hypothesis $A \backslash A(\omega) \subseteq L_{i}\left(a, \omega^{\prime}\right) \cap A\left(\omega^{\prime}\right) \cup A \backslash A\left(\omega^{\prime}\right) \subseteq L_{i}^{s}\left(a, \omega^{\prime}\right)$ for all $i$. It follows that $L_{i}^{s}(a, \omega) \subseteq L_{i}^{s}\left(a, \omega^{\prime}\right)$ for all $i$. By $s$-monotonicity $s\left(a, \omega^{\prime}\right)=a \in F\left(\omega^{\prime}\right)$.

(Sufficiency) Let $F$ satisfy WU and GCC. Let $a \in F(\omega)$. Assume that $L_{i}^{s}(a, \omega) \subseteq$ $L_{i}^{s}\left(a, \omega^{\prime}\right)$ for all $i$. We first prove by contradiction that $a \in A\left(\omega^{\prime}\right)$ : assume $a \notin A\left(\omega^{\prime}\right)$. If $A\left(\omega^{\prime}\right) \subseteq A(\omega)$, by WU, there exist an agent $i$ and $b \in A\left(\omega^{\prime}\right)$ such that $a R_{i}(\omega) b$. Then $a R_{i}^{s}(\omega) b$, and $b P_{i}^{s}\left(\omega^{\prime}\right) a$, because $a$ is not feasible at $\omega^{\prime}$, which yields a contradiction. Now let $A\left(\omega^{\prime}\right) \nsubseteq A(\omega)$. In this case, let $i$ be any agent and let $b \in A\left(\omega^{\prime}\right) \backslash A(\omega)$. Then $a P_{i}^{s}(\omega) b$, and $b P_{i}^{s}\left(\omega^{\prime}\right) a$, because $a$ is not feasible at $\omega^{\prime}$, which yields a contradiction. So far we have established that $a \in F(\omega) \cap A\left(\omega^{\prime}\right)$. We next prove by contradiction that $L_{i}(a, \omega) \cap A\left(\omega^{\prime}\right) \subseteq L_{i}\left(a, \omega^{\prime}\right)$ and $A\left(\omega^{\prime}\right) \backslash A(\omega) \subseteq L_{i}\left(a, \omega^{\prime}\right)$ for all $i \in I$. If this holds we can conclude by GCC. Assume first that $L_{i}(a, \omega) \cap A\left(\omega^{\prime}\right) \nsubseteq L_{i}\left(a, \omega^{\prime}\right)$ for some agent $i$. Let $b \in\left[L_{i}(a, \omega) \cap A\left(\omega^{\prime}\right)\right] \backslash L_{i}\left(a, \omega^{\prime}\right)$. We have $a R_{i}^{s}(\omega) b$ and $b P_{i}^{s}\left(\omega^{\prime}\right) a$, which yields 
a contradiction. Now assume that, for some agent $i, A\left(\omega^{\prime}\right) \backslash A(\omega) \nsubseteq \nsubseteq L_{i}\left(a, \omega^{\prime}\right)$. Let $b \in\left[A\left(\omega^{\prime}\right) \backslash A(\omega)\right] \backslash L_{i}\left(a, \omega^{\prime}\right)$. We have $a P_{i}^{s}(\omega) b$ and $b P_{i}^{s}\left(\omega^{\prime}\right) a$, which yields a contradiction.

\section{Non-rewarding reversion functions: applications}

In this section, we apply the findings of the previous sections to withholding in exchange economies, bargaining and taxation methods. In these environments, natural form of punishment are available. In pure exchange economies endowments can be confiscated, in bargaining problems agents can be reverted to the disagreement point. If such forms of reversion are used, agents weakly prefer any feasible allocation to any infeasible one. The difference with the generalized severe reversion function is that some agents might be indifferent between a feasible allocation and an infeasible one. If such reversion functions are non-rewarding, WU and GCC are not only necessary but also sufficient for monotonicity.

Lemma 1 Let $h$ be a non-rewarding reversion function such that, for every $\omega \in \Omega$, for all $a \in A(\omega)$ and for all $b \notin A(\omega), a R_{i}(\omega) h(b, \omega)$ for all $i \in I$. The SCR $F$ is $h$-monotonic if and only if it is monotonic under generalized severe reversion.

Proof It suffices to prove that if $F$ is monotonic under generalized severe reversion then it is $h$-monotonic. The reverse implication follows from Proposition 1. Let $s$ denote the generalized severe reversion function and assume that $F$ is $s$-monotonic. Let $a \in F(\omega)$ and assume that $L_{i}^{h}(a, \omega) \subseteq L_{i}^{h}\left(a, \omega^{\prime}\right)$ for all $i$. As $h$ is non-rewarding, $a \in A\left(\omega^{\prime}\right)$. It follows that $L_{i}^{h}\left(a, \omega^{\prime}\right)=L_{i}^{s}\left(a, \omega^{\prime}\right)=L_{i}\left(a, \omega^{\prime}\right) \cap A\left(\omega^{\prime}\right) \cup A \backslash A\left(\omega^{\prime}\right)$. As $a \in A(\omega), L_{i}^{h}(a, \omega)=L_{i}^{s}(a, \omega)=L_{i}(a, \omega) \cap A(\omega) \cup A \backslash A(\omega)$. The claim follows from the hypothesis that $F$ is $s$-monotonic.

\subsection{Exchange economies: withholding}

Reversion functions might be rewarding in this environment. For instance, starting from an allocation in which agents are given goods that they scarcely care about, if the endowment of one good is reduced by, say, $1 \%$ and renegotiation does not entail any cost, it is possible to renegotiate this allocation in a way in which all of them are better off. The non-rewarding condition is plausible here if we assume that renegotiation is sufficiently costly, e.g., delay, transaction costs, etc., for at least one agent.

Consider an exchange economy with $n$ agents and $K$ goods. We assume that agents' preferences do not vary but that endowments do. Let $u_{i}$ be a utility function that represents agent $i$ 's preferences. We assume that $u_{i}$ is strictly increasing, continuous and quasi-concave. Let $\Omega_{i} \subseteq \mathbf{R}_{+}^{K}$ be the set of agent $i$ 's possible endowments and let $\prod_{i=1}^{n} \Omega_{i}$. We consider a form of punishment suited to the environment. We assume that, should an infeasibility arise, agents' endowments are confiscated and a small punishment is assigned to each of them. Then, the allocation set contains the set of the balanced net transfers, the generalized punishment point and $C$, which represents the situation where agents' endowment are confiscated: 
$A=\left\{x \in \mathbf{R}^{K \times n}: \sum_{s=1}^{n} x_{s}=0\right\} \cup\{C, G\}$. For all $\omega \in \Omega$ the feasible set is $A(\omega)=$ $\{x \in A: 0 \leq x+\omega\} \cup\{C, G\}{ }^{8}$ Then $A\left(\omega^{\prime}\right) \subseteq A(\omega)$ if and only if $\omega^{\prime} \leq \omega$. In order to describe preferences on net transfers, note that the utility agent $i$ gets from transfer $x_{i}$ when her endowment is $\omega_{i}$ is $u_{i}\left(x_{i}+\omega_{i}\right)$. Thus, the utilities that agents derive from allocations are state dependent even if preferences are not. For every $i \in I$, for every $\omega \in \Omega$, let $u_{i}(C, \omega)=0$ and $u_{i}(G, \omega)=u_{i}(0)-\varepsilon$, for some $\varepsilon>0$. Finally, let $h$ such that $h(x, \omega)=C$ for all $x \notin A(\omega)$.

Remark 2 The reversion function $h$, as defined above, is non-rewarding. Let us check that it satisfies (i) and (ii) in Definition 5. First, assume that $x \in A(\omega) \backslash A\left(\omega^{\prime}\right)$. In order to prove that both (i) and (ii) hold, it suffices to show that there exists $y \in$ $A\left(\omega^{\prime}\right)$ such that $x R_{i}(\omega) h(y, \omega)$ and $y P_{i}\left(\omega^{\prime}\right) h\left(x, \omega^{\prime}\right)$ for some $i$. Consider the case $A\left(\omega^{\prime}\right) \backslash A(\omega) \neq \emptyset$. For all $y \in A\left(\omega^{\prime}\right) \backslash A(\omega)$ we have $y_{i} \neq-\omega_{i}^{\prime}$ for some $i$, then $x R_{i}(\omega) h(y, \omega)$ and $y P_{i}\left(\omega^{\prime}\right) h\left(x, \omega^{\prime}\right)$ because preferences are strictly increasing. The remaining case is when $A\left(\omega^{\prime}\right) \subseteq A(\omega)$, then $\omega^{\prime} \leq \omega$. Let $i$ such that $x_{i} \neq-\omega_{i}$. Let $y \in A\left(\omega^{\prime}\right)$ such that $-\omega_{i}^{\prime}<y_{i} \leq x_{i}$. Then $x R_{i}(\omega)$ y and $y P_{i}\left(\omega^{\prime}\right) h\left(x, \omega^{\prime}\right)$ because preferences are strictly increasing. In order to complete the proof, we have to show that (ii) holds for every $x \in A(\omega) \cap A\left(\omega^{\prime}\right)$. First, consider the case $A\left(\omega^{\prime}\right) \backslash$ $A(\omega) \neq \emptyset$. In order to prove the claim, it suffices to show that there exists $j$ such that $A\left(\omega^{\prime}\right) \backslash A(\omega) \nsubseteq L_{j}\left(x, \omega^{\prime}\right)$. Indeed, for any $y \in A\left(\omega^{\prime}\right) \backslash A(\omega), y \notin L_{j}\left(x, \omega^{\prime}\right)$, we have $x R_{j}(\omega) h(y, \omega), y P_{j}\left(\omega^{\prime}\right) x$. As $\omega^{\prime}\left\lfloor\leq \omega, \omega_{i k}^{\prime}>\omega_{i k}\right.$ for some agent $i$ and for some good $k$. First, assume $x_{i k}+\omega_{i k}^{\prime}>0$. Set $y_{i k}=-\omega_{i k}^{\prime}$, and set $y_{j k}=$ $x_{j k}+x_{i k}+\omega_{i k}^{\prime}>x_{j k}$ for some $j \neq i$. Set $y_{r l}=x_{r l}$ if $r \neq i, j$ or if $l \neq k$. Transfer $y$ is feasible at $\omega^{\prime}$ but not at $\omega$ and preferences are strictly increasing so $x R_{j}(\omega) h(y, \omega), y P_{j}\left(\omega^{\prime}\right) x$. Now assume $x_{i k}+\omega_{i k}^{\prime}=0$. Let $j \neq i$ such that $x_{j k}>0$. Let $\delta$ be such that $\delta<\min \left\{x_{j k}, \omega_{i k}^{\prime}-\omega_{i k}\right\}$. Set $y_{i k}=x_{i k}+\delta$, and set $y_{j k}=x_{j k}-\delta$. Set $y_{r l}=x_{r l}$ if $r \neq i, j$ or if $l \neq k$. Transfer $y$ is feasible at $\omega^{\prime}$ but not at $\omega$ and preferences are strictly increasing so $x R_{i}(\omega) h(y, \omega), y P_{i}\left(\omega^{\prime}\right) x$. The remaining case is when $A\left(\omega^{\prime}\right) \subseteq A(\omega)$ then $\omega^{\prime} \leq \omega$ and let $x \in A\left(\omega^{\prime}\right)$. If $y P_{i}^{h}\left(\omega^{\prime}\right) x$ and $x R_{i}^{h}(\omega) y$ then $y \in A\left(\omega^{\prime}\right)$ so (ii) holds.

In this environment the no veto condition holds emptily when $h$ and the generalized severe reversion function are used. From Lemma 1 it follows that a SCR is $h$-implementable if and only if it satisfies WU and GCC.

Let us translate conditions WU and GCC into this framework. First consider WU. It suffices to consider only endowments $\omega, \omega^{\prime}$ such that $\omega^{\prime} \leq \omega$, because, as observed above, $A\left(\omega^{\prime}\right) \subseteq A(\omega)$ if and only if $\omega^{\prime} \leq \omega$. Then WU amounts to the following condition:

Condition $\alpha$ For all $\omega, \omega^{\prime} \in \Omega$ such that $\omega^{\prime} \leq \omega$, if $a \in F(\omega) \backslash A\left(\omega^{\prime}\right)$ there exists $i$ such that $u_{i}\left(\omega_{i}+a_{i}\right) \geq u_{i}\left(\omega_{i}-\omega_{i}^{\prime}\right)$.

Observe that if $\left(0, \omega_{i}\right) \subseteq \Omega_{i}$ for all $i$ then Condition $\alpha$ only requires the SCR to be individually rational for at least one agent. It is a very weak requirement and it is obviously satisfied by many SCR, e.g., any Pareto efficient or any individually rational SCR.

\footnotetext{
${ }^{8}$ For $x, y \in \mathbf{R}^{n}$ we write $x \leq y$ if $x_{i} \leq y_{i}$ for all $i, x<y$ if $x_{i} \leq y_{i}$ for all $i$ and $x_{j}<y_{j}$ for at least one $j$.
} 
Stronger requirements are imposed by GCC. In this case it also suffices to consider only endowments $\omega, \omega^{\prime} \in \Omega$ such that $\omega^{\prime} \leq \omega$. Indeed, assume that $\omega^{\prime} \not \leq \omega$. In this case $A\left(\omega^{\prime}\right) \backslash A(\omega) \nsubseteq L_{j}\left(a, \omega^{\prime}\right)$ for some $j$ (see Remark 2). So, if $\omega^{\prime} \not \leq \omega$ GCC holds emptily. It follows that GCC is satisfied if and only if the following condition holds: Condition $\boldsymbol{\beta}$ For all $\omega, \omega^{\prime} \in \Omega$ such that $\omega^{\prime} \leq \omega$, if $a \in F(\omega) \cap A\left(\omega^{\prime}\right)$ and $a \notin F\left(\omega^{\prime}\right)$ there exists $i$ and $x \in A\left(\omega^{\prime}\right)$ such that

$$
\begin{aligned}
& u_{i}\left(\omega_{i}+a_{i}\right) \geq u_{i}\left(\omega_{i}+x_{i}\right) \\
& u_{i}\left(\omega_{i}^{\prime}+a_{i}\right)<u_{i}\left(\omega_{i}^{\prime}+x_{i}\right)
\end{aligned}
$$

Consider now a social choice function $f: \Omega \rightarrow A$ such that $f(\omega) \in A(\omega)$ for all $\omega \in \Omega$. For every $\omega \in \Omega$ let $f_{i}(\omega)$ be the transfer that $f$ assigns to player $i$ at $\omega$, which is $f(\omega)=\left(f_{1}(\omega), \ldots, f_{n}(\omega)\right)$. Let us compare our conditions with Hong (1998), Corollary 1, p. $216 .^{9}$ She showed that $f$ is implementable by a collection of state dependent mechanisms if and only if, for all $\omega, \omega^{\prime} \in \Omega$, the following condition is satisfied:

$$
\omega \geq \omega^{\prime} \Longrightarrow u_{i}\left(\omega_{i}+f_{i}\left(\omega_{i}\right)\right) \geq u_{i}\left(\omega_{i}-\omega_{i}^{\prime}\right) \text { for all } i \in I
$$

Our Condition $(\alpha)$ is weaker than condition $(\mathrm{H})$. Indeed, in this case $(\alpha)$ requires only that $u_{i}\left(\omega_{i}+f_{i}(\omega)\right) \geq u_{i}\left(\omega_{i}-\omega_{i}^{\prime}\right)$ for at least one $i$ when $f(\omega) \notin A\left(\omega^{\prime}\right)$.

Note that our condition depends on the fact that each agent cannot simply retain part of her endowment. She has to make it compatible with other agents' messages. But our Condition $(\beta)$ is not implied by Condition $(\mathrm{H})$. Assume for instance that $f(\omega) \in A\left(\omega^{\prime}\right)$. We have $f_{i}(\omega) \geq-\omega_{i}^{\prime}$ for all $i$ so $u_{i}\left(\omega_{i}+f_{i}(\omega)\right) \geq u_{i}\left(\omega_{i}-\omega_{i}^{\prime}\right)$ for all $i$ because all $u_{i}$ are increasing. Then (H) imposes no restrictions on $f\left(\omega^{\prime}\right)$. If the translations by $\omega-\omega^{\prime}$ of all agents' indifference curves through $\omega^{\prime}+f(\omega)$ are strictly above all agents' indifference curves through $\omega+f(\omega)$, condition $(\beta)$ implies $f(\omega)=f\left(\omega^{\prime}\right)$. Formally, if $\left.\bigcap_{i=1}^{n}\left\{y: u_{i}\left(\omega_{i}^{\prime}+f_{i}(\omega)\right)\right)=u_{i}\left(y_{i}\right)\right\} \subseteq$ $\bigcap_{i=1}^{n}\left\{y: u_{i}\left(y_{i}+\omega_{i}-\omega_{i}^{\prime}\right)>u_{i}\left(\omega_{i}+f_{i}(\omega)\right)\right\}$, condition $(\beta)$ imposes that $f(\omega)=$ $f\left(\omega^{\prime}\right)$.

Two of Hong's assumptions make our approaches different: (i) Hong assumes that players cannot exaggerate their endowment and that they can be punished for the message they send and not only for the allocation they intend to obtain if such an allocation is not feasible; and (ii) Hong gives each player the power to retain part of her endowment. We assume that players can collectively cheat the planner through the mechanism by asking for a feasible allocation in which some agents retain a part of their endowment.

\footnotetext{
${ }^{9}$ In Hong's framework a mechanism is a pair $\left(\{M(\omega)\}_{\omega \in \Omega}, g\right) . M(\omega)=\prod_{i \in I} M_{i}\left(\omega_{i}\right)$ is the message space at state $\omega$ and $g$ is a function, $g: M \rightarrow A$ where $M=\prod_{i \in I} M_{i}, M_{i}=\bigcup_{\omega_{i} \in \Omega_{i}} M\left(\omega_{i}\right)$. The mechanism must be feasible at every state of the world, which is $g(M(\omega)) \subseteq A(\omega)$ for all $\omega$. Agents are subject to no exaggeration assumption: when her endowment is $\omega_{i}$, agent $i$ must send messages within $\bigcup_{\omega_{i}^{\prime} \leq \omega_{i}} M_{i}\left(\omega_{i}\right)$. Such an assumption implies that agents use different message spaces at different states of the world.
} 
Let us first study the implementation of the Constrained Walrasian SCR. ${ }^{10}$ The allocation $a \in A(\omega)$ is a Constrained Walrasian Allocation (CWA) at $\omega$ if there exists $p \in \mathbf{R}_{+}^{K}$ such that $a \in \arg \max \left\{u_{i}\left(\omega_{i}+x_{i}\right): p x_{i} \leq 0, x \in A(\omega)\right\}$ for all $i$. Such a $p$ is said to be an equilibrium price supporting $a$ at $\omega$. Let $\mathrm{CW}(\omega)$ denote the set of CWA at $\omega$.

Proposition 3 Let $\Omega_{i}=\left(0, \bar{\omega}_{i}\right)$ for all $i$, for some $\bar{\omega}_{i} \in(0, \infty)$. Then the Constrained Walrasian SCR is implementable in Nash Equilibrium if $h$ or the generalized severe reversion function are used.

Proof Under our assumptions, $C W(\omega)$ is not empty for all $\omega \in \Omega$. In order to prove the claim it suffices to show that $C W$ satisfies Condition $\beta$. Let $\omega^{\prime} \leq \omega, a \in C W(\omega) \cap$ $A\left(\omega^{\prime}\right)$ and $a \notin C W\left(\omega^{\prime}\right)$. Let $p$ be an equilibrium price at $\omega$. Then there exist $x \in$ $A\left(\omega^{\prime}\right)$ with $u_{i}\left(\omega^{\prime}+x_{i}\right)>u_{i}\left(\omega^{\prime}+a_{i}\right)$ and $p x_{i} \leq 0$ for some $i . A\left(\omega^{\prime}\right) \subseteq A(\omega)$ so $x \in\left\{x \in A(\omega), p x_{i} \leq 0\right\}$. From the definition of $C W$ it follows that $u_{i}\left(\omega_{i}+a_{i}\right) \geq$ $u_{i}\left(\omega_{i}+x_{i}\right)$. Then $C W$ satisfies Condition $\beta$.

A similar result holds for fixprice equilibria. Here we use the definition of Younès equilibrium (Younès 1975), though there are other concepts of fixprice equilibria, such as Dréze's (1975) equilibrium (see also Grandmont and Laroque 1976), which includes restrictions on sales and Benassy's equilibrium (1975), which uses rationing schemes. Silvestre (1982) proved that the three concepts are equivalent.

Definition 9 Given a vector of prices $p$, an allocation $x^{*}$ is a Constrained Younès Equilibrium at $\omega$ if it satisfies:

(i) $\sum_{i=1}^{n} x_{i}^{*}=0$

(ii) For all $i x_{i}^{*}+\omega_{i}$ maximizes $u_{i}$ on the set $\left\{z_{i}-\omega_{i}: z_{i} \in \mathbf{R}_{+}^{K}, \min \left\{x_{i j}^{*}, 0\right\} \leq\right.$ $\left.z_{i j}-\omega_{i j} \leq \max \left\{x_{i j}^{*}, 0\right\}, p\left(z_{i}-\omega_{i}\right) \leq 0\right\} \cap A(\omega)$

(iii) There exist no pair of consumers $(i, h)$, a commodity $k$ and a real number $\varepsilon>0$ such that $u_{i}\left(\omega_{i}+x_{i}^{*}+\varepsilon a^{k}\right)>u_{i}\left(\omega_{i}+x_{i}^{*}\right)$ and $u_{h}\left(\omega_{h}+x_{h}^{*}-\varepsilon a^{k}\right)>u_{i}\left(\omega_{h}+x_{h}^{*}\right)$, where $a^{k}$ is defined by $a_{1}^{k}=-p_{k}, a_{k}^{k}=1$ and $a_{t}^{j}=0$ for $t \neq 1, k$.

We obtain the following result using the same argument as in Proposition 3:

Remark 3 The constrained Younès Equilibrium correspondence is implementable in Nash Equilibrium if $h$ or the generalized severe reversion function are used.

\subsection{Bargaining with unknown utility possibility set}

Let us now consider non-cooperative implementation of cooperative solution concepts (Dagan and Serrano 1998; Naeve 1999). A bargaining problem is a pair $(U, v)$

10 The Walrasian Correspondence defined by WC $(\omega)=\arg \max \left\{u_{i}\left(\omega_{i}+x_{i}\right): p x_{i} \leq 0\right\}$ is not implementable in Nash Equilibrium under a generalized severe reversion function for the same reasons that prevent the standard Nash implementability of WC. 
where $U \subseteq \mathbf{R}_{+}^{n}$ is the utility possibility set and $v \in U$ is the disagreement point. We assume that $U$ is convex, closed, with a non-empty interior and comprehensive (i.e., $u \in U$ and $u^{\prime} \leq u, u^{\prime} \in \mathbf{R}_{+}^{n}$ implies $u^{\prime} \in U$ ). For each bargaining problem $(U, v)$, let $U_{v}=\{u \in U: u \geq v\}$ be bounded. The Nash Bargaining Solution (NBS) is defined as $\operatorname{NBS}(U, v)=\arg \max _{u \in U_{v}} \prod_{i=1}^{n}\left(u_{i}-v_{i}\right)$. Let $\operatorname{NBS}(U, v)_{i}$ be the utility awarded to $i$ by the NBS. For further reference we notice that this solution is characterized by the following properties: strong efficiency, individual rationality, scale covariance, symmetry, and independence of irrelevant alternatives.

We consider $U_{v} \cup\{G\}$ as the feasible set of $(U, v)$. We consider a non-rewarding reversion function suited to the situation and assume that all infeasible allocations are reverted to the disagreement point. Let $h$ denote such reversion function, which we will call non-severe because $h(a, \omega) \neq G$ for all $a \in A$ and all $\omega \in \Omega$. Agent $i$ 's reverted preferences at $(U, v)$ are described by

$$
\begin{gathered}
\left.u_{i}^{h}(u,(U, v))\right)=u_{i} \quad \text { if } u \in U_{v} \\
u_{i}^{h}(u,(U, v))=v_{i}, \quad \text { otherwise. }
\end{gathered}
$$

The reversion function is non-rewarding. Condition (i) is satisfied because any infeasible allocation is reverted to the disagreement point and any feasible vector of utilities different from the disagreement point allocates to at least one agent a utility level strictly higher than the disagreement level. Condition (ii) is satisfied with $b=c$ and $i=j$. Assume that $a R_{i}^{h}(\omega) h(b, \omega)$ but $h\left(b, \omega^{\prime}\right) P_{i}^{h}\left(\omega^{\prime}\right) h\left(a, \omega^{\prime}\right)$ for some $i$. Then $b \in A\left(\omega^{\prime}\right)$, otherwise $b$ would be reverted to the disagreement point and $h\left(a, \omega^{\prime}\right) R_{i}\left(\omega^{\prime}\right) h\left(b, \omega^{\prime}\right)$.

We first notice that if the disagreement point is not known by the planner, NBS fails to satisfy GCC: Let $n=2$ and let $U=\left\{x \in \mathbf{R}_{+}^{2}: x_{1}^{2}+x_{2}^{2} \leq 1\right\}$. Let $v=(0,0)$ and let $v^{\prime}=\left(\frac{\sqrt{2}}{2}, 0\right)$. Then $\operatorname{NBS}(U, v)=\left(\frac{\sqrt{2}}{2}, \frac{\sqrt{2}}{2}\right) \in U_{v^{\prime}} \subseteq U_{v}$ but $\operatorname{NBS}\left(U, v^{\prime}\right) \neq$ NBS $\left(U, v^{\prime}\right)$. Thus, according to Proposition 1 , NBS is not implementable in NE by any non-rewarding reversion function. ${ }^{11}$

11 This result agrees with the findings obtained by Serrano and Vohra (1997) in a different framework. A different interpretation of preferences on the utility possibility set may lead to more permissive results. One can interpret them as a measure of agents' satisfaction with respect to the disagreement point. Let $u_{i}(u,(U, v))=u_{i}-v_{i}$. Then the preferences induced by $h$ are

$$
\begin{aligned}
& u_{i}^{h}(u,(U, v))=u_{i}-v_{i} \quad \text { if } \quad u \in U_{v} \\
& u_{i}^{h}(u,(U, v))=0 \quad \text { otherwise }
\end{aligned}
$$

Observe that $u_{i}^{h}(u,(U, v))=u_{i}^{h}(u-v,(U-v, 0))$. The reader can easily check that from the translation invariance property of the NBS, the analysis of the problem with unknown disagreement point amounts to the previous situation with the disagreement point fixed and known at 0 . In this case, applying Proposition 4 yields a positive result. 
The Kalai-Smorodinski solution does not satisfy GCC even with a fixed disagreement point. Proposition 1 implies that it cannot be implemented in NE by any nonrewarding reversion function.

Instead, when the disagreement point is known, the NBS satisfies both GCC and WU, as the reader can easily verify. From Lemma 1 it follows that it is $h$-monotonic. However, we cannot conclude that the NBS is implementable because it does not satisfy the no veto condition and the Maskin Theorem requires at least three agents (see Remark 1).

We prove the result directly by using the characterizations developed by Moore and Repullo (1990) (MR from now on).

Proposition 4 Let $n \geq 2$. The Nash Bargaining Solution is implementable in Nash Equilibrium with the non-severe reversion function if the disagreement point $v$ is known.

Proof Let $x=\operatorname{NBS}(U, v)$. We prove that the sets $\mathbf{R}_{+}^{n},\left\{L_{i}^{h}(x,(U, v))\right\}_{i \in I}$, where $x=\operatorname{NBS}(U, v)$, satisfy condition $\mu$ in MR (p. 1088). For every $\left(U^{\prime}, v\right)$ they satisfy condition (i) (MR p. 1088) because the NBS is $h$-monotonic, so it suffices to show that they satisfy conditions (ii) and (iii) (MR p. 1088) for every $\left(U^{\prime}, v\right)$. We start by proving that they satisfy (ii). Let $x=\operatorname{NBS}(U, v)$. Let $i \in I$ and consider a bargaining problem $\left(U^{\prime}, v\right)$. Let $u \in L_{i}^{h}(x,(U, v))$ such that, with preferences reverted at $\left(U^{\prime}, v\right), u$ is maximal in $L_{i}^{h}(x,(U, v))$ for agent $i$ and is maximal in $\mathbf{R}_{+}^{n}$ for all agents different from $i$, which is $u_{i} \geq u_{i}^{\prime}$, for all $u^{\prime}=\left(u_{i}^{\prime}, u_{-i}^{\prime}\right) \in L_{i}^{h}(x,(U, v))$ and $u_{j}=\max \left\{u_{j}^{\prime}: \exists u_{-j}^{\prime},\left(u_{j}^{\prime}, u_{-j}^{\prime}\right) \in U_{v}^{\prime}\right\}$ for all $j \neq i$. To prove (ii) we have to show that $u=\operatorname{NBS}\left(U^{\prime}, v\right)$. If $u=x$ the claim follows from $h$-monotonicity. Then, assume $u \neq x$. We prove by contradiction that $x=\operatorname{NBS}(U, v)$. Assume that $u \neq \operatorname{NBS}(U, v)$. It must be the case that $u$ is feasible at $\left(U^{\prime}, v\right)$ otherwise, with preferences reverted at $\left(U^{\prime}, v\right)$, all agents different from $i$ would strictly prefer some point in the interior of $U_{v}^{\prime}$ to $u$. Furthermore, $u$ lies on the boundary of $U_{v}^{\prime}$. As NBS is efficient, we have $x=\operatorname{NBS}(U, v)_{i}>u_{i}$. Consider the segment $S$ joining $x=\operatorname{NBS}(U, v)$ and $u$. $S \subseteq U_{v}^{\prime}$ because $U_{v}^{\prime}$ is convex and if $t \in S \backslash\{u\}, t_{i}>u_{i}$. It follows that $S \backslash\{u\} \subseteq U_{v}$, otherwise for all $t \notin U_{v}, t \in S \backslash\{u\}, t \in L_{i}^{h}(x,(U, v))$ and $t_{i}>u_{i}$, in contradiction with the hypothesis that $u$ is maximal for $i$ in $L_{i}^{h}(x,(U, v))$ with preferences reverted at $\left(U^{\prime}, v\right)$. In particular $\operatorname{NBS}(U, v) \in U_{v}$ and $u \in U_{v}$ because $U_{v}$ is closed. Furthermore, $\operatorname{NBS}(U, v) \neq x$ and $x \notin U_{v^{\prime}}^{\prime}$, because $u$ is maximal in $L_{i}^{h}(x,(U, v))$ under reverted preferences for $i, u \neq x$ and NBS is efficient. $U_{v}$ is convex, $\operatorname{NBS}(U, v) \in U_{v}$ and $x \notin U_{v}^{\prime}$ so $S^{\prime}=\left\{u^{\prime} \in U_{v}: x_{i} \geq u_{i}^{\prime}\right\} \cap S \backslash\{u\}$ is not empty. Let $u^{\prime} \in S^{\prime}$, then $u \in L_{i}^{h}(x,(U, v)) \cap U_{v}^{\prime}$, but $u_{i}^{\prime}>u_{i}$, in contradiction with the hypothesis that $u$ is maximal for $i$ in $L_{i}^{h}(x,(U, v))$ with preferences reverted at $\left(U^{\prime}, v\right)$. Then $u=\operatorname{NBS}(U, v)$. In order to prove (iii) we have to show that if $u$ is maximal in $\mathbf{R}_{+}^{n}$ for all agents when preferences reverted at $\left(U^{\prime}, v\right)$ which is $u_{j}=\max \left\{u_{j}^{\prime}: \exists u_{-j}^{\prime},\left(u_{j}^{\prime}, u_{-j}^{\prime}\right) \in U_{v}^{\prime}\right\}$ for all $j$, then $u=\operatorname{NBS}(U, v)$. The claim follows from the efficiency of the NBS. From Theorem 1 (MR p.1088) follows that the NBS is $h$-implementable if $n \geq 3$.

Finally, for $n=2$, we show that the sets $\mathbf{R}_{+}^{2},\left\{L_{i}^{h}(\operatorname{NBS}(U, v),(U, v))\right\}_{i=1,2}$, satisfy condition $\mu 2$ in MR (p.1091) for all $(U, v)$. Above we have proved that they satisfy 
(i), (ii) and (iii). Property (iv) (MR p. 1091) holds emptily because of the disagreement point. From Theorem 2 (MR p. 1091) follows that the NBS is $h$-implementable when $n=2$.

\subsection{Taxation}

A taxation problem is a pair $(x, T) \in R_{+}^{n} \times R_{+}$, where $x$ is the vector of taxable incomes and $T$ is the total amount to be collected such that $\sum_{i=1}^{n} x_{i} \geq T$ (Dagan et al. 1999). A tax allocation $t$ for the taxation problem $(x, T)$ is a vector in $R_{+}^{n}$ such that $t \leq x$ and $\sum_{i=1}^{n} t_{i}=T$. A taxation rule is a function $f$ which assigns a tax allocation to each taxation problem. We assume that the planner knows the amount to be collected, $T$, but does not know the vector of taxable incomes $x$. Let $S^{n}(T)=\left\{t \in R_{+}^{n}: \sum_{i=1}^{n} t_{i}=T\right\}$ be the set of tax allocations that collect $T$. Let $\Omega^{n}(T)=\left\{x \in R_{+}^{n}: \sum_{i=1}^{n} x_{i}>T\right\}$ be the set of the states of the world. Let $T^{n}(x)=$ $T^{n}(x, T)=\left\{t \in R_{+}^{n}: 0 \leq t \leq x, \sum_{i=1}^{n} t_{i}=T\right\} \cup\{G\}$ be the set of feasible tax allocations at $x$. Each agent's preferences only depend on her after tax income and are strictly increasing. Then we can write $u_{i}(t, x)=x_{i}-t_{i}$ for each $x \in \Omega^{n}(T)$ and for each $t \in T^{n}(x, T) \backslash\{G\}$.

WU always holds. We now characterize GCC in this environment.

Proposition 5 A taxation rule satisfies $G C C$ if and only if $f(x)=f\left(x^{\prime}\right)$ for all $x, x^{\prime}$ such that $f(x) \leq x^{\prime} \leq x$.

Proof The necessity of the condition follows directly from the definition of GCC, because if $x^{\prime} \leq x$ then $T^{n}\left(x^{\prime}\right) \subseteq T^{n}(x)$. We next prove the sufficiency of the condition. Let $t=\bar{f}(x) \in T^{n}\left(x^{\prime}\right)$. First consider $x, x^{\prime} \in \Omega^{n}(T)$ such that $x^{\prime} \leq x$. Then $T^{n}\left(x^{\prime}\right) \subseteq T^{n}(x)$. This implies that $f(x) \leq x^{\prime} \leq x$. It follows that $f(x)=f\left(x^{\prime}\right)$. Then, the condition required by GCC is satisfied. Now consider the case in which $x_{j}<x_{j}^{\prime}$ for at least one $j$. The condition $T^{n}\left(x^{\prime}\right) \backslash T^{n}(x) \subseteq L_{i}\left(t, x^{\prime}\right)$ for all $i$ is equivalent to $T^{n}\left(x^{\prime}\right) \backslash T^{n}(x) \subseteq \bigcap_{i=1}^{n} L_{i}\left(t, x^{\prime}\right)=\{t\} \cup\{G\}$. It is impossible in this case because the set $T^{n}\left(x^{\prime}\right) \backslash T^{n}(x) \supseteq\left\{t \in R_{+}^{n}: 0 \leq t \leq x^{\prime}, x_{j}<t_{j} \leq x_{j}^{\prime}, \sum_{i=1}^{n} t_{i}=T\right\}$ is not empty and has the cardinality of the continuum. In this case, the condition required by GCC holds emptily.

Under the no exaggeration assumption, any taxation rule can be implemented in NE (see Dagan et al. 1999). It is no longer true in our framework. Consider the proportional taxation rule, defined as

$$
f_{i}(x)=\frac{x_{i}}{\sum_{i=1}^{n} x_{i}} T, \quad \text { for } i=1, \ldots, n
$$

Such a rule is not implementable in Nash equilibrium under any non rewarding reversion function. We prove the claim by contradiction. Assume that $f$ is implementable. Let $x^{*} \in \Omega^{n}(T)$. Observe that $0<f_{i}\left(x^{*}\right)<x_{i}^{*}$ for all $i$. From the continuity of $f$, it follows that there is a neighborhood $U$ of $x^{*}$ that $0<f_{i}(x)<x_{i}^{*}$ for $i=1, \ldots, n$, if $x$ belongs to $U$. Let $x \in U \cap\left\{x: x^{*} \leq x\right\}$ which is not empty. We 
have $0<f(x)<x^{*} \leq x$ for all such $x$. As $f$ is monotonic, Proposition 5 implies that $f(x)=f\left(x^{*}\right)$, which yields a contradiction.

More in general, let $f$ be a continuous taxation rule such that for some $x, 0<$ $f_{i}(x)<x_{i}$ for all $i$. If $f$ is continuous but not constant whenever incomes are larger than proposed taxes for $x$, it cannot be implemented in our framework (the proof is available upon request).

The difference between our result and the result obtained by Dagan et al. (1999) stems from the fact that no exaggeration of incomes is possible in their approach. In their model an agent is not allowed to exaggerate anyone's income and not only her own income as in Hong (1998). In our case agents are punished only if their message leads to an infeasible allocation. To explain the argument, consider the mechanism used by Dagan et al. to implement a feasible taxation rule $f$. At state $x$, a message for player $i$ is a vector of incomes $y^{i}=\left(y_{1}^{i}, \ldots, y_{n}^{i}\right)$ such that $y^{i} \leq x$ (the no exaggeration assumption) and $\sum_{j=1}^{n} y_{j}^{i} \geq T$. Payments are assigned according to $f\left(y^{*}\right)$, where $y_{i}^{*}=\max \left\{y_{i}^{j}: 1 \leq j \leq n\right\}$. Every player has to report the income of all agents without exaggerating them. Agent $i$ pays the tax she should pay if her real income was the maximum income some player reported for her. Dagan et al. prove that the messages $m_{i}=x$ for all $i$, constitute a Nash equilibrium at state $x$. In our setup players may profit from exaggerating other agents' income if it leads to a feasible allocation so such equilibria cannot be sustained. Consider a continuous taxation rule such that $0<f_{i}(x)<x_{i}$ for all $i$. Assume that the contribution of agent $i$ is a decreasing function of other agents' incomes. ${ }^{12}$ Let $y$ such that $y_{i}=x_{i}$ and $y_{j}^{i}=x_{j}+\varepsilon$ for all $j \neq i$, where $\varepsilon>0$ is such that $f(y) \leq x$. Then, when all other agents reveal the true vector of incomes, agent $i$ can profitably deviate by sending message $y$ and paying $f_{i}(y)<f_{i}(x)$. She would not be punished because $f(y)$ is feasible at $x$.

\section{Conclusions}

This paper presents a new approach to dealing with the implementation problem when feasible sets are state dependent. It is based on the idea that feasibility is restored by a process that is independent of the mechanism and that reflects how agents renegotiate infeasible allocations into feasible ones or the working of institutions designed to cope with infeasibility. We have presented the class of non-rewarding reversion functions and we have focused our attention in those that we call generalized severe. We have shown that this class implements the maximal set of social choice rules and thus, despite of its extreme form, it provides an easy test for implementability in our framework. We have not provided a characterization of non-rewarding reversion functions but this topic should be subject of further research. Finally, we have studied the implementation in Nash equilibrium of social choice rules in exchange economies, bargaining problems and taxation methods, and we have compared our results with those obtained in earlier literature. In some cases, like bargaining, generalized severe reversion functions are close to what the literature assumed but in others they are too

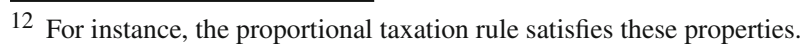


extreme. Thus, it would be good to know which SCR can be implemented with less drastic reversion functions. This topic is also left for future research.

A feature of the traditional approach of implementation when feasible sets are state dependent is that it requires a collection of state dependent mechanisms, which stands in contrast to the case when preferences are state dependent. This distinction differs vividly from our intuition on how markets cope with infeasible allocations, namely that the sign of excess demand entirely determines the adjustment irrespective of the cause of infeasibility. Thus, our approach may offer a better understanding of market mechanisms than the traditional one, though the traditional approach is better suited to deal with topics such as withholding of endowments. Our approach can be generalized to deal with this case by introducing uncertainty in the renegotiation process or by writing the mechanism as an argument in the reversion function. These two extensions are easy to write, but require completely new analytical methods and therefore are left for future research.

Acknowledgments We would like to thank Felipe Balmaceda Carmen Beviá, Roberto Serrano, Asher Wolinski and the audiences at the III Workshop of Social Choice (Málaga), the Economic Theory Seminar in Bilbao, the Workshop in Microeconomic Theory at Rutgers University; as well as the associate editor and a referee for their very useful comments. Both authors acknowledge financial support from CAICYT under project SEJ2005-06167/ECON and from Fundación BBVA. The second author acknowledge financial support from FONDECYT under project 11080132.

\section{References}

Amorós P (2004) Nash implementation and uncertain renegotiation. Games Econ Behav 49:424-434

Atlamaz M, Klaus B (2007) Manipulation via endowments in exchange markets with indivisible goods. Soc Choice Welf 28:1-18

Benassy J-P (1975) Neokeynesian disequilibrium theory in a monetary economy. Rev Econ Stud 52: $503-524$

Dagan N, Serrano R (1998) Invariance and randomness in the Nash program for coalitional games. Econ Lett 58:43-49

Dagan N, Serrano R, Volij O (1999) Feasible implementation of taxation methods. Rev Econ Design 4: $57-72$

Dréze J (1975) Existence of an exchange equilibrium under price rigidities. Int Econ Rev 16:301-320

Grandmont JM, Laroque G (1976) On temporary Keynesian equilibria. Rev Econ Stud 43:53-68

Hong L (1995) Nash implementation in production economies. Econ Theory 5:401-417

Hong L (1996) Bayesian implementation in exchange economies with state dependent feasible sets and private information. Soc Choice Welf 13:433-444

Hong L (1998) Feasible Bayesian implementation with state dependent feasible sets. J Econ Theory 80: 201-221

Hurwicz L, Maskin E, Postlewaite A (1995) Feasible Nash implementation of social choice rules when the designer does not know endowments or production set. In: Ledyard J (ed) The economics of informational decentralization: complexity, efficiency and stability. Kluwer Academic Publishing, Dordrecht, pp 367-433

Jackson M (2001) A crash course in implementation theory. Soc Choice Welf 18:655-708

Jackson M, Palfrey T (2001) Voluntary implementation. J Econ Theory 98:1-25

Maskin E (1999) Nash equilibrium and welfare optimality. Rev Econ Stud 66:23-38

Maskin E, Moore J (1999) Implementation and renegotiation. Rev Econ Stud 66:39-56

Moore J, Repullo R (1990) Nash implementation: a full characterization. Econometrica 58:1083-1089

Naeve J (1999) Nash implementation of the Nash bargaining solution using intuitive message spaces. Econ Lett 62:23-28

Postlewaite A (1979) Manipulation via endowments. Rev Econ Stud 46:255-262 
Repullo R (1987) A simple proof of Maskin's theorem on Nash implementation. Soc Choice Welf 4:39-41 Roemer JE (1996) Theories of distributive justice. Harvard University Press, Cambridge

Serrano R (1997) A comment on the Nash program and the theory of implementation. Econ Lett 55:203-208 Serrano R, Vohra R (1997) Non cooperative implementation of the Core. Soc Choice Welf 14:513-525

Silvestre J (1982) Fixprice analysis in exchange economies. J Econ Theory 26:28-58

Tian G (1993) Implementing Lindahl allocations by a withholding Mechanism. J Math Econ 22:163-179

Tian G, Li Q (1995) On Nash-implementation in the presence of withholding. Games Econ Behav 9: $222-233$

Younès Y (1975) On the role of money in the process of exchange and the existence of a non-Walrasian equilibrium. Rev Econ Stud 42:489-502 